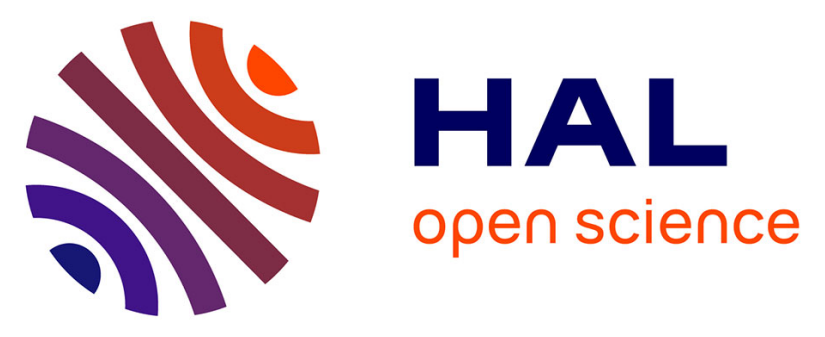

\title{
Single-Atom Addressing in Microtraps for Quantum-State Engineering using Rydberg Atoms
}

Henning Labuhn, Sylvain Ravets, Daniel Barredo, Lucas Béguin, Florence Nogrette, Thierry Lahaye, Antoine Browaeys

\section{- To cite this version:}

Henning Labuhn, Sylvain Ravets, Daniel Barredo, Lucas Béguin, Florence Nogrette, et al.. SingleAtom Addressing in Microtraps for Quantum-State Engineering using Rydberg Atoms. Physical Review A: Atomic, molecular, and optical physics [1990-2015], 2014, 90, pp.023415. 10.1103/PhysRevA.90.023415 . hal-01010696

\section{HAL Id: hal-01010696 https://hal.science/hal-01010696}

Submitted on 16 Nov 2015

HAL is a multi-disciplinary open access archive for the deposit and dissemination of scientific research documents, whether they are published or not. The documents may come from teaching and research institutions in France or abroad, or from public or private research centers.
L'archive ouverte pluridisciplinaire HAL, est destinée au dépôt et à la diffusion de documents scientifiques de niveau recherche, publiés ou non, émanant des établissements d'enseignement et de recherche français ou étrangers, des laboratoires publics ou privés. 


\title{
Single-atom addressing in microtraps for quantum-state engineering using Rydberg atoms
}

\author{
Henning Labuhn, Sylvain Ravets, Daniel Barredo, Lucas Béguin, Florence Nogrette, Thierry Lahaye, and Antoine Browaeys \\ Laboratoire Charles Fabry, UMR 8501, Institut d'Optique, CNRS, Univ Paris Sud 11, \\ 2 avenue Augustin Fresnel, 91127 Palaiseau cedex, France
}

(Received 19 June 2014; published 22 August 2014)

\begin{abstract}
We report on the selective addressing of an individual atom in a pair of single-atom microtraps separated by $3 \mu \mathrm{m}$. Using a tunable light shift, we render the selected atom off-resonant with a global Rydberg excitation laser which is resonant with the other atom, making it possible to selectively block this atom from being excited to the Rydberg state. Furthermore we demonstrate the controlled manipulation of a two-atom entangled state by using the addressing beam to induce a phase shift onto one component of the wave function of the system, transferring it to a dark state for the Rydberg excitation light. Our results are an important step towards implementing quantum information processing and quantum simulation with large arrays of Rydberg atoms.
\end{abstract}

DOI: 10.1103/PhysRevA.90.023415

PACS number(s): 37.10.Gh, 32.80.Ee, 03.67.Bg

Cold neutral atoms are a promising platform for quantum computation and quantum simulation [1]. Their weak interactions in the ground state lead to long coherence times. Using highly excited Rydberg states allows one to switch on and off the strong interactions that are necessary for engineering many-body quantum states [2]. For many of those experiments it is desirable to confine single atoms at well-defined positions separated by a few micrometers, which can be achieved, e.g., using arrays of optical tweezers [3]. Another requirement is the selective manipulation of individual atoms in the ensemble. This can be done by applying static field gradients, or a laser beam focused to one single trap site, which induces a frequency shift at the targeted site. Such techniques have been demonstrated with trapped ions [4-6] and neutral atoms in optical lattices [7-13].

In previous work $[14,15]$, we have demonstrated quantumstate engineering with single atoms held in two and three optical microtraps, by using the Rydberg blockade mechanism with global excitation of the atoms. Extending these studies to a larger number of atoms and to wider classes of quantum states requires extra tools. A step towards this goal was our recent demonstration of single-atom trapping in large arrays of optical microtraps with arbitrary geometries [3]. Combined with global excitation, this already opens the possibility to generate interesting multiatom entangled states, such as the $W$ state $|W\rangle=(|r g g \cdots g\rangle+|g r g \cdots g\rangle+\cdots+$ $|g g g \cdots r\rangle) / \sqrt{N}$, where $|g\rangle(|r\rangle)$ corresponds to the ground (Rydberg) state. However, single-site addressing is needed to engineer other classes of quantum states. For instance, the realization of the collective controlled-NOT (CNOT) gate of Ref. [16] that can be used to create the Greenberger-HorneZeilinger state $|\mathrm{GHZ}\rangle=(|g g \cdots g\rangle+|r r \cdots r\rangle) / \sqrt{2}$, requires the singling out of one control atom whose state determines the state of the remaining, target, atoms.

Here we demonstrate the selective addressing of one single ${ }^{87} \mathrm{Rb}$ atom among two atoms held in microtraps separated by $3 \mu \mathrm{m}$, by shining a tightly focused, red-detuned $850-\mathrm{nm}$ laser beam on it. This addressing beam induces a frequency shift on the ground state of the atom, while leaving its Rydberg states nearly unaffected. This differential light shift thus makes the addressed atom off-resonant with the Rydberg excitation laser, which is resonant for the other atom. This article is organized as follows. We first briefly describe the implementation of the addressing beam, and characterize its size and depth in situ using a single atom. We then perform a global Rydberg excitation in the presence of the addressing beam, and observe nearly perfect suppression of excitations for the addressed atom. Finally, we use the addressing beam to perform a controlled local operation on one atom, coherently transferring the symmetric entangled state $(|r g\rangle+|g r\rangle) / \sqrt{2}$ to the antisymmetric, dark state $(|r g\rangle-|g r\rangle) / \sqrt{2}$.

Our experimental setup, schematically shown in Fig. 1(a), has been described previously $[3,14,15]$. We use a spatial light modulator (SLM) to create two microtraps, separated by a distance of $3 \mu \mathrm{m}$ in the focal plane of a high-numericalaperture (NA) aspherical lens. The traps, each with a $1 / e^{2}$ radius of about $1 \mu \mathrm{m}$ and a depth of $U_{0} \approx h \times 20 \mathrm{MHz}$, are focused in an ${ }^{87} \mathrm{Rb}$ magneto-optical trap (MOT). Due to fast light-assisted collisions, we trap only either zero or one atom per trap [17], and trigger the experiment on the presence of one atom in each trap. The temperature of the atoms in the traps is approximately $50 \mu \mathrm{K}$. We coherently couple the ground state $|g\rangle=\left|5 S_{1 / 2}, F=2, m_{f}=2\right\rangle$ to the Rydberg state $|r\rangle=$ $\left|n D_{3 / 2}, m_{j}=3 / 2\right\rangle$ (with $n$ in the range 50-100) via a twophoton transition, with the wavelengths of the excitation lasers being 795 and $474 \mathrm{~nm}$. During the excitation, of duration $\tau$, the traps are switched off to avoid extra broadening arising from the shot-to-shot fluctuations of the light shift due to the random positions of the atoms in the traps. The detuning from the intermediate state $\left|5 P_{1 / 2}, F=2, m_{f}=2\right\rangle$ is $2 \pi \times 740 \mathrm{MHz}$. After the excitation pulse, we measure the states of both atoms. Repeating the experiment about 100 times, we reconstruct the populations $P_{i j}$ of the two-atom states $|i j\rangle$, where $i$ and $j$ can take the values $g$ and $r$.

The $1 / e^{2}$ radii of the lasers used for Rydberg excitation are $100 \mu \mathrm{m}$ for the $795-\mathrm{nm}$ beam, and $18 \mu \mathrm{m}$ for the $474-\mathrm{nm}$ beam. This configuration prevents the direct addressing of a single trap. To achieve single-site addressability, we thus induce an extra light shift on the ground state of the atom at the targeted site, to selectively control the Rydberg excitation. As a fast (i.e., on microsecond time scales) reconfiguration cannot be achieved with the SLM, we superimpose a second, independently controlled 850-nm laser beam onto the trapping beam. Orthogonal polarizations and a frequency difference of 
(a)
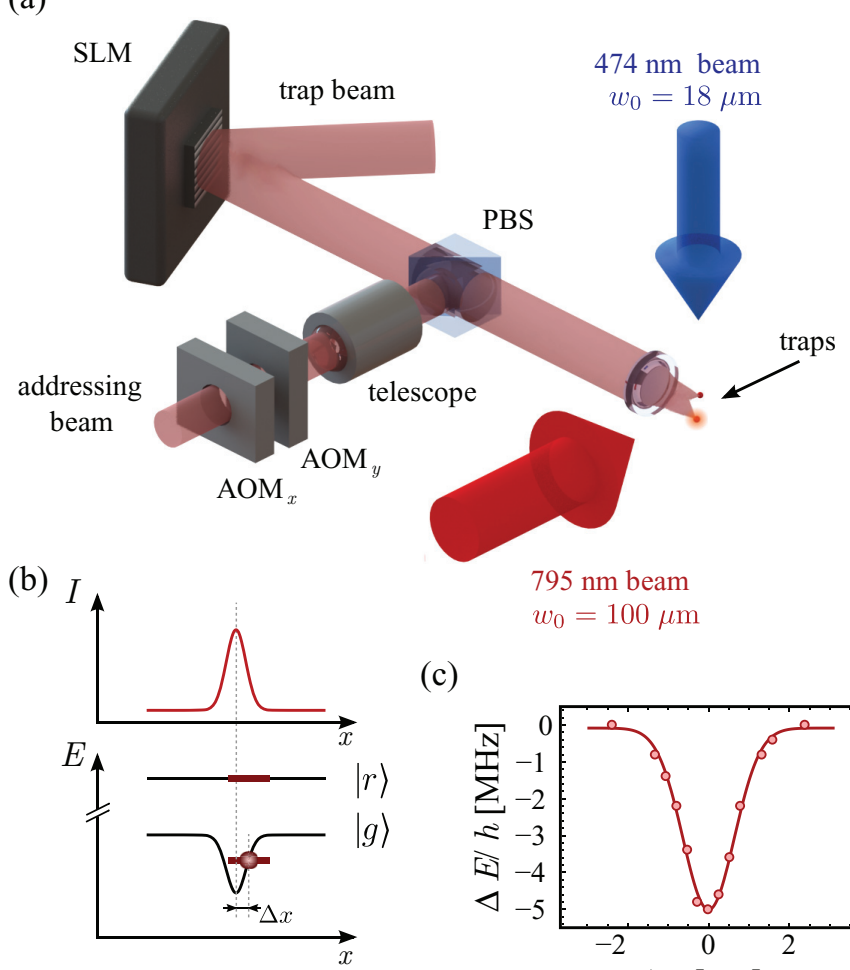

(c)

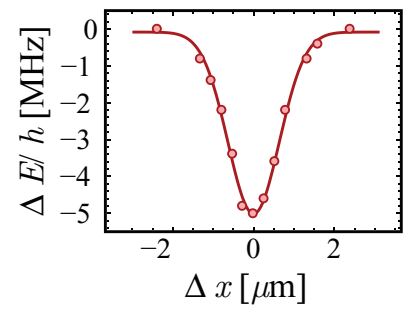

FIG. 1. (Color online) (a) Sketch of the experimental setup. The two microtraps are created by a red-detuned $850-\mathrm{nm}$ laser beam on which an appropriate phase is imprinted using a spatial light modulator (SLM), and focused by a high-NA aspheric lens in a MOT. The addressing beam is superimposed onto the trap beam by a polarizing beam-splitter cube (PBS), and focused down on the targeted atom by the same aspheric lens. The two perpendicular AOMs can be used for precise dynamical $x-y$ positioning of the addressing beam. A telescope is used to conjugate the AOM plane with the aspheric lens, to avoid clipping when the addressing beam is deflected. (b) The light shift $\Delta E$ of the ground state of the targeted single atom is directly proportional to the intensity $I$ of the addressing beam at the position $x$ of the atom. (c) Measured light shift $\Delta E$ as a function of the distance $\Delta x$ between the addressing beam and the targeted trap, yielding a $1 / e^{2}$ radius of the addressing beam of $w_{0} \simeq 1.3 \mu \mathrm{m}$.

about $200 \mathrm{MHz}$ prevent interference between the trapping and addressing beams. The addressing beam has a $1 / e^{2}$ radius of $w_{0} \simeq 1.3 \mu \mathrm{m}$ in the focus, slightly larger than the trap size. This choice results from a trade-off between two opposite requirements, namely, minimizing alignment sensitivity and inhomogeneous light shifts (which favors a large $w_{0}$ ) and minimizing cross-talk (which implies choosing a small $w_{0}$ ). For a perfectly Gaussian beam with $w_{0} \simeq 1.3 \mu \mathrm{m}$, one expects theoretically that if one atom is addressed by a light shift of $10 \mathrm{MHz}$, the second atom $3 \mu \mathrm{m}$ away experiences a light shift of only $0.2 \mathrm{kHz}$, which is negligible as compared to the other relevant frequencies in the experiment. An electrooptic modulator enables fast (about $10 \mathrm{~ns}$ ) switching of the addressing beam. In addition, two acousto-optic modulators (AOMs) can be used for dynamical $x-y$ positioning of the addressing beam with respect to the targeted trap.
In a first experiment, we measure the intensity profile of the addressing beam in situ by performing Rydberg spectroscopy on a single atom. For different positions $\Delta x$ of the addressing beam with respect to the targeted atom, we scan the frequency of the Rydberg excitation lasers. As mainly the ground state experiences a light shift $\Delta E$ proportional to the addressing beam intensity, the resonance frequency for Rydberg excitation is shifted by $\Delta E$ [see Fig. 1(b)]. Figure 1(c) shows the measured light shift as a function of $\Delta x$. A Gaussian fit gives a $1 / e^{2}$ radius $w_{0}=1.3 \pm 0.1 \mu \mathrm{m}$. The residual light shift experienced by the nearby atom $3 \mu \mathrm{m}$ away is below the resolution of our experiment.

We observe that for large light shifts, the probability of losing an atom during the sequence increases. We attribute this effect to the following: due to the finite temperature, the atom never sits exactly at the intensity maximum of the addressing beam. The fast switching on and off of the addressing beam thus imparts kinetic energy to the atom. This increase in kinetic energy becomes more and more important as the intensity of the addressing beam gets larger. For large enough intensities in the addressing beam, this effect thus increases the probability for the atom to leave the trapping region during the experiment. However, for light shifts below $40 \mathrm{MHz}$, this loss probability remains below $1 \%$, and is thus negligible.

We now perform a Rydberg blockade experiment with two single atoms in order to demonstrate single-site addressability (Fig. 2). In Ref. [18], site-resolving excitation beams were used to demonstrate blockade with two atoms separated by $10 \mu \mathrm{m}$. Here, we use a global excitation scheme in combination with the addressing beam, and obtain similar results, albeit with a distance between the atoms of only $3 \mu \mathrm{m}$. For both atoms, the ground state $|g\rangle$ is coupled to the Rydberg state $|r\rangle=\left|59 D_{3 / 2}\right\rangle$ with a Rabi frequency $\Omega \simeq 2 \pi \times 1 \mathrm{MHz}$ [Fig. 2(a)]. If the atoms were independent, they would both undergo Rabi oscillations between $|g\rangle$ and $|r\rangle$ with the Rabi frequency $\Omega$. The strong dipole-dipole interaction $U_{\mathrm{dd}}$ between the Rydberg states forbids a double excitation of the atoms if $U_{\mathrm{dd}} \gg \hbar \Omega$. This condition is largely fulfilled for the parameters chosen here: the interaction energy of two atoms in $\left|59 D_{3 / 2}\right\rangle$, separated by $3 \mu \mathrm{m}$, is approximately $h \times 300 \mathrm{MHz}$. We thus excite only the superposition state $|s\rangle=\left(|r g\rangle+e^{i \boldsymbol{k} \cdot \boldsymbol{r}}|g r\rangle\right) / \sqrt{2}$, whose coupling to the two-atom ground state $|g g\rangle$ is $\sqrt{2} \Omega[18,19]$ (here, $\boldsymbol{k}$ is the vector sum of the wave vectors of the excitation lasers, and $\boldsymbol{r}$ is the position of atom 2 with respect to atom 1). This results in $P_{r g}$ and $P_{g r}$ oscillating between 0 and $1 / 2$ with a frequency $\sqrt{2} \Omega$, as can be seen in Fig. 2(b). Another signature of the blockade is the suppression of double excitation $P_{r r} \simeq 0$ [see bottom panel in Fig 2(b)].

If we shine the addressing beam on atom 2 , we observe a strong suppression of the excitation probability for the states $|g r\rangle$ and $|r r\rangle$ [see Fig. 2(c)], as atom 2 is never excited to the Rydberg state $|r\rangle$. At the same time, atom 1 shows Rabi oscillations between $|g\rangle$ and $|r\rangle$ with the single-atom Rabi frequency $\Omega$. The small residual excitation probability of atom 2 that we observe is fully accounted for by the errors in our state detection [15], meaning that cross-talk between the two traps is negligible.

Finally, we show that we can also use the addressing beam to directly manipulate a two-atom quantum state. Without any addressing, the excitation to the state $|r r\rangle$ is completely 
(a)

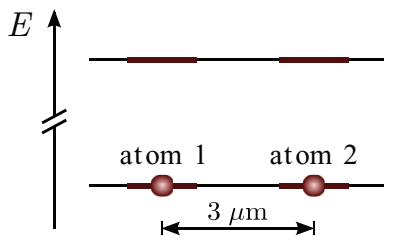

(b)
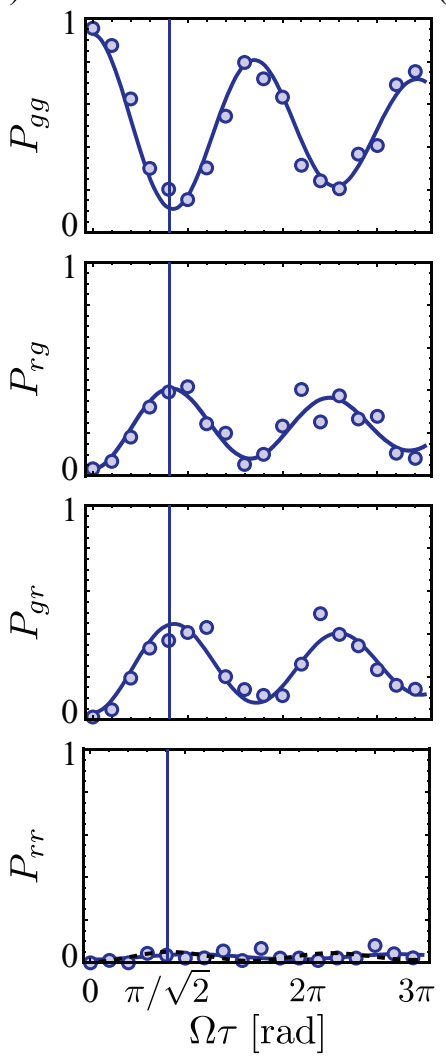

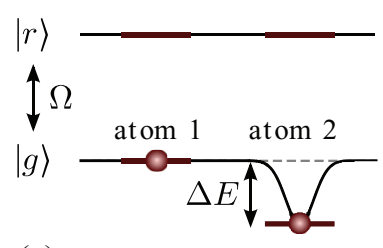

(c)
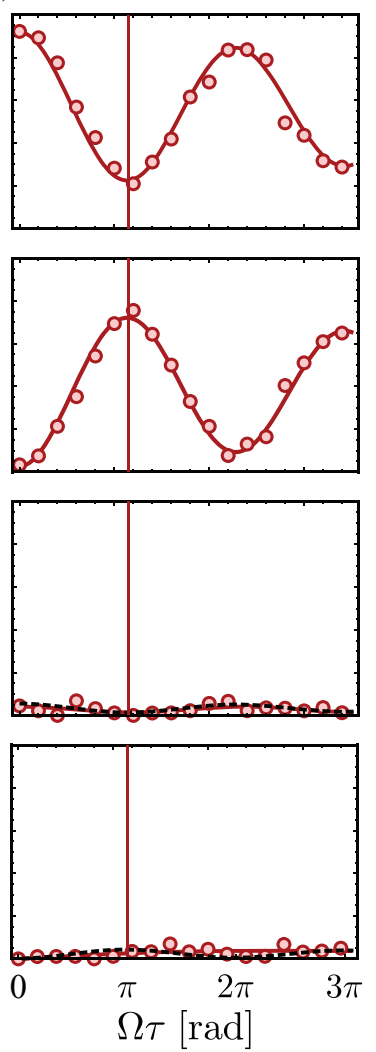

FIG. 2. (Color online) (a) Two atoms, separated by $3 \mu \mathrm{m}$, are illuminated by light that resonantly couples the ground state $|g\rangle$ to $|r\rangle=\left|59 D_{3 / 2}\right\rangle$ with the single-atom Rabi frequency $\Omega$. The time evolution of the populations of the two-atom states $|g g\rangle,|g r\rangle,|r g\rangle$, and $|r r\rangle$ are shown, (b) without any addressing and (c) with atom 2 addressed with a light shift of $\Delta E \simeq h \times 10 \mathrm{MHz}$. Solid lines are fits by damped sine curves. The vertical solid lines mark the pulse areas $\Omega \tau$ corresponding to a $\pi$ pulse for the nonaddressed case (blue) and the addressed case (red). The black dashed lines show the expected measured populations for a perfect blockade of atom 2, taking into account state-detection errors.

suppressed in the Rydberg blockade regime $\left(U_{\mathrm{dd}} \gg \hbar \Omega\right)$. By applying an excitation pulse of duration $\pi /(\sqrt{2} \Omega)$ we thus prepare the atoms in the state $|\psi(0)\rangle=\left(|g r\rangle+e^{i k \cdot r}|r g\rangle\right) / \sqrt{2}$. We then illuminate atom 2 with the addressing beam [Fig. 3(a)]. Its energy is shifted by $\Delta E$ when in the ground state, while its Rydberg state remains unaffected [see Fig. 2(a)]. After a time $T$ the state of the system has therefore evolved to

$$
|\psi(T)\rangle=\frac{1}{\sqrt{2}}\left(|g r\rangle+e^{-i \Delta E T / \hbar} e^{i \boldsymbol{k} \cdot \boldsymbol{r}}|r g\rangle\right) .
$$

The antisymmetric dark state $\left|\psi\left(T_{\pi}\right)\right\rangle=\left(|g r\rangle-e^{i \boldsymbol{k} \cdot \boldsymbol{r}}|r g\rangle\right) /$ $\sqrt{2}$ (with $T_{\pi}=\pi \hbar / \Delta E$ ) is not coupled to the ground state (a)

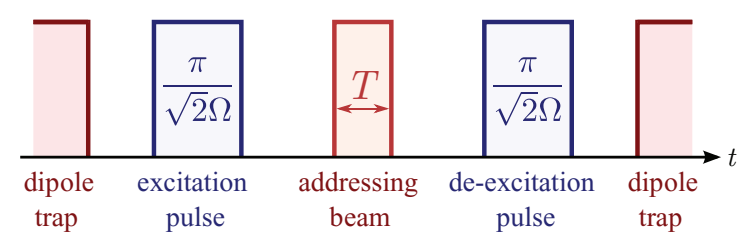

(b)

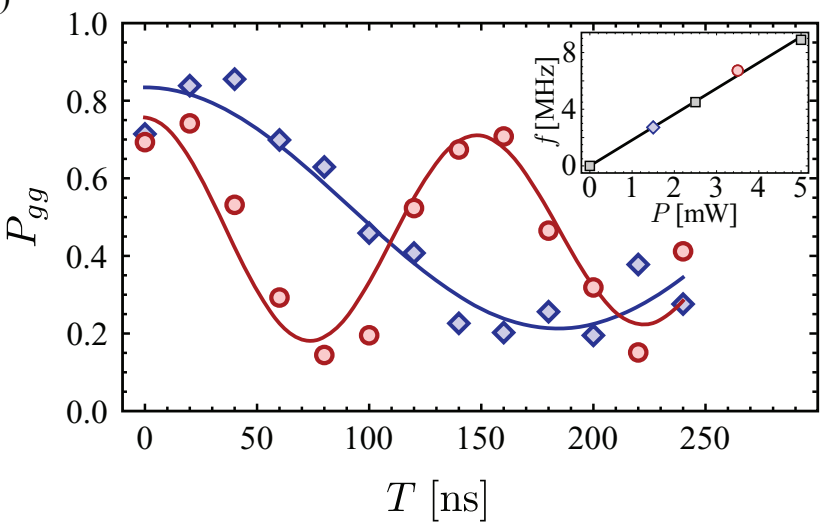

FIG. 3. (Color online) (a) Pulse sequence for the phase manipulation: while the dipole trap is switched off, the atoms are excited to the state $|s\rangle=\left(|g r\rangle+e^{i \boldsymbol{k} \cdot \boldsymbol{r}}|r g\rangle\right) / \sqrt{2}$. The addressing beam induces a light shift $\Delta E$ on the ground state of atom 2, thus changing the relative phase evolution between $|g r\rangle$ and $|r g\rangle$. This is followed by a global deexcitation pulse. (b) Population of the two-atom ground state $|g g\rangle$ after the deexcitation pulse, as a function of the addressing pulse length $T$, for a laser power in the addressing beam $P=$ $1.5 \mathrm{~mW}$ (blue diamonds) and $P=3.5 \mathrm{~mW}$ (red circles). Solid lines are fits by damped sine curves of frequency $f$. Inset: oscillation frequency $f$ as a function of the power $P$ of the addressing beam, showing the expected linear dependence. For this experiment we use the Rydberg state $\left|82 D_{3 / 2}\right\rangle$.

$|g g\rangle$. The probability of deexciting the atoms to $|g g\rangle$ is thus expected to oscillate between 0 and 1 with a frequency $f=\Delta E / h$.

Figure 3(b) shows the probability $P_{g g}$ of deexciting the atoms back to $|g g\rangle$ versus the duration $T$ of the addressing pulse. We observe the expected oscillation of the final groundstate population $P_{g g}$. Due to the finite Rydberg excitation efficiency (about $90 \%$ for our parameters), we measure a contrast of the oscillations that is lower than 1 . In addition, the finite temperature of the atoms in the experiment leads to a small motion of the atoms during the sequence, implying that (i) the phase $\boldsymbol{k} \cdot \boldsymbol{r}$ imprinted by the excitation pulse is not exactly canceled out by the deexcitation pulse [20]; and (ii) the light shift $\Delta E$ experienced by atom 2 fluctuates from shot to shot. Averaged over many runs, both effects lead to a decreased contrast and a finite damping of the observed oscillations. To take these effects into account, we fitted the data with a damped sine curve of the form $P_{g g}(T)=A+B \exp (-\gamma t) \cos (2 \pi f T)$, with the oscillation frequency $f$ and the damping rate $\gamma$ as adjustable parameters. Repeating the experiment for different powers of the addressing beam, we obtain the expected linear dependence of $f$ with the applied light shift on the atom [see inset of Fig. 3(b)]. This demonstrates our ability to perform some controlled local operations on qubits in a quantum register. 
In conclusion, we have shown that we can selectively prevent one single atom in a pair of single-atom traps from being resonant with Rydberg excitation lasers, with no measurable cross-talk with a neighboring atom as close as $3 \mu \mathrm{m}$. We also demonstrated the use of the addressing beam to perform a local operation in a system of two atoms. Our scheme is easily scalable to a larger number of traps. These techniques will prove useful for a variety of applications in quantum simulation and quantum information processing with Rydberg atoms. For instance, they open the possibility of selectively addressing a single qubit in a larger ensemble, e.g., as a control atom for realizing collective quantum gates [16], or to excite a single atom to a different Rydberg state, allowing the study of the transfer of excitations along a Rydberg chain [21].

We thank Yvan Sortais for helpful advice about the optical design. We acknowledge financial support by the EU [ERC Stg Grant ARENA, AQUTE Integrating Project, FET-Open Xtrack Project HAIRS, and EU Marie-Curie Program ITN COHERENCE Grant No. FP7-PEOPLE-2010-ITN-265031 (H.L.)], by DGA (L.B.), and by Région Île-de-France (LUMAT and Triangle de la Physique, LAGON Project).
[1] I. Bloch, J. Dalibard, and S. Nascimbène, Nat. Phys. 8, 267 (2012).

[2] M. Saffman, T. G. Walker, and K. Mølmer, Rev. Mod. Phys. 82, 2313 (2010).

[3] F. Nogrette, H. Labuhn, S. Ravets, D. Barredo, L. Béguin, A. Vernier, T. Lahaye, and A. Browaeys, Phys. Rev. X 4, 021034 (2014).

[4] H. C. Nägerl, D. Leibfried, H. Rohde, G. Thalhammer, J. Eschner, F. Schmidt-Kaler, and R. Blatt, Phys. Rev. A 60, 145 (1999).

[5] H. Häffner, W. Hänsel, C. F. Roos, J. Benhelm, D. Chek-al-kar, M. Chwalla, T. Körber, U. D. Rapol, M. Riebe, P. O. Schmidt, C. Becher, O. Gühne, W. Dür, and R. Blatt, Nature 438, 643 (2005).

[6] C. Monroe and J. Kim, Science 339, 1164 (2013).

[7] R. Dumke, M. Volk, T. Müther, F. B. J. Buchkremer, G. Birkl, and W. Ertmer, Phys. Rev. Lett. 89, 097903 (2002).

[8] M. Saffman, Opt. Lett. 29, 1016 (2004).

[9] M. Karski, L. Förster, J.-M. Choi, A. Steffen, N. Belmechri, W. Alt, D. Meschede, and A. Widera, New J. Phys. 12, 065027 (2010).

[10] C. Weitenberg, M. Endres, J. F. Sherson, M. Cheneau, P. Schauß, T. Fukuhara, I. Bloch, and S. Kuhr, Nature (London) 471, 319 (2011).

[11] M. Schlosser, S. Tichelmann, J. Kruse, and G. Birkl, Quantum Inf. Process. 10, 907 (2011).
[12] T. Fukuhara, A. Kantian, M. Endres, M. Cheneau, P. Schauß, S. Hild, D. Bellem, U. Schollwöck, T. Giamarchi, C. Gross, I. Bloch, and S. Kuhr, Nat. Phys. 9, 235 (2013).

[13] J. H. Lee, E. Montano, I. H. Deutsch, and P. S. Jessen, Nat. Commun. 4, 2027 (2013).

[14] L. Béguin, A. Vernier, R. Chicireanu, T. Lahaye, and A. Browaeys, Phys. Rev. Lett. 110, 263201 (2013).

[15] D. Barredo, S. Ravets, H. Labuhn, L. Béguin, A. Vernier, F. Nogrette, T. Lahaye, and A. Browaeys, Phys. Rev. Lett. 112, 183002 (2014).

[16] M. Müller, I. Lesanovsky, H. Weimer, H. P. Büchler, and P. Zoller, Phys. Rev. Lett. 102, 170502 (2009).

[17] Y. R. P. Sortais, H. Marion, C. Tuchendler, A. M. Lance, M. Lamare, P. Fournet, C. Armellin, R. Mercier, G. Messin, A. Browaeys, and P. Grangier, Phys. Rev. A 75, 013406 (2007).

[18] E. Urban, T. A. Johnson, T. Henage, L. Isenhower, D. D. Yavuz, T. G. Walker, and M. Saffman, Nat. Phys. 5, 110 (2009).

[19] A. Gaëtan, Y. Miroshnychenko, T. Wilk, A. Chotia, M. Viteau, D. Comparat, P. Pillet, A. Browaeys, and P. Grangier, Nat. Phys. 5, 115 (2009).

[20] T. Wilk, A. Gaëtan, C. Evellin, J. Wolters, Y. Miroshnychenko, P. Grangier, and A. Browaeys, Phys. Rev. Lett. 104, 010502 (2010).

[21] S. Wüster, C. Ates, A. Eisfeld, and J. M. Rost, Phys. Rev. Lett. 105, 053004 (2010). 